\title{
Concurrent Multifunction Transmit and Receive Applications with Dynamic Filtering
}

\author{
Trevor Snow $\left({ }^{*}, * *\right)$, Eric J. Naglich $\left({ }^{* *}\right)$, and William J. Chappell $\left({ }^{* *}\right)$ \\ (*) NSWC Crane, 300 Highway 361, Crane, IN 47522 \\ (**) Department of Electrical and Computer Engineering, Purdue University \\ 465 Northwestern Ave, West Lafayette, IN 47907 \\ ideasrf@ecn.purdue.edu
}

(812) 219-9138

\begin{abstract}
This paper demonstrates a concept for transmitting on one set of elements of a phased array while concurrently receiving on another set at a separate frequency. Cases are analyzed using measured data from an $8 x 8$ microstrip stacked patch panel array operating at 3$3.8 \mathrm{GHz}$, and relative coupling levels from interfering elements to receive elements are characterized over various scan angles for two array configurations - side-by-side contiguous blocks and for interleaved elements at separate frequencies. It is shown that an optimum transmit frequency exists that minimizes coupled power to receiving elements in the array for a given scan angle. Additional isolation between elements is achieved with a tunable bandpassbandstop filter cascade that, when paired with a highlinearity low-noise amplifier, reduces interference from transmitting elements to within the receiver analog-todigital converter's (ADC) dynamic range, such that receiving elements can operate concurrently next two $25 \mathrm{~W}$ transmitting elements.
\end{abstract}

\section{INTRODUCTION}

Multi-function radar combines many radar sensing operations into a common aperture and processing framework. What once may have been multiple separate and completely independent sensing operations on different and widely separated antennas can now be performed by a single phased array antenna system tied to common data processing resources. For a multifunctional radar in an environment where the majority of radar sensing tasks are low priority such as friendly target tracking, all tasks are allocated enough time and radar resources without impacting required performance. When a radar is presented with many high priority targets, radar resources are thinned out due to tracking of the highest priority targets, and other tasks are sacrificed. This demonstrates a need for compressing the time sharing schedule for a radar.

Copyright information:

U.S. Government work not protected by U.S. copyright
To prevent one function from interfering with the operations of other functions, isolation between different functions is required and often achieved by serially time-sharing aperture and processing resources [1]. In the simplest case, pulses are sent out and the subsequent function may not start transmitting until return pulses arrive from the maximum range covered by the original pulse. Subsequent pulses may belong to a coherent pulse train for a single task, or different tasks at different scan angles.

In a common radar transmitter/receiver configuration, coupled power from the transmit pulse would saturate the receive chain or even damage it, so the receiver is protected with some combination of a circulator, switch, or a limiter. Because of this limitation on the receiver, range blind zones exist. This is especially an issue at ranges close in to the radar, as the minimum sensing range is related to the pulse width and level of clutter around the radar[2]. These blind zones limit the ability to detect targets near the radar antenna face, and when using a multifunctional scan, blind regions are induced within the middle of the range of detection. Employing a staggered pulse-repetition frequency (PRF) can alter where these blind zones occur from pulse to pulse and also improves maximum unambiguous range [3]. However, in low peak-power radars that rely on long pulses for improved sensitivity, staggering the PRF can only go so far to reduce blind zones.

Alternatively, functions may be separated in frequency. Some phased array concepts use elements widely separated in frequency such that elements for one function are far outside the operating bandwidth of other elements in the array [4]. Waveform/frequency diversity and beam forming diversity can additionally help resolve range and Doppler ambiguities and separate radar functions. Even with frequency separation from pulse to pulse or between functions, self-interference from the transmitter requires that the receiver is blanked during transmit periods.

This paper presents a system architecture using tunable filters in a phased array that would allow for concurrent transmission while receiving at a different frequency within the radar band. The amount of coupling as a function of scan angle is presented and the impact of this coupling on 
tunable front-end filtering components is determined as a function of scan angle. In the conceptual architecture, an array with large contiguous adjacent blocks of microstrip patch elements operating at separate frequencies is analyzed. The same array is reanalyzed but with interleaved elements where every other element is operating at one of two different frequencies. These configurations are both examined for their coupling properties between elements, as higher coupling would increase interference between transmitting and receiving elements, requiring more isolation from the tunable filters.

\section{System CONFIGURATION}

In recently emerging advanced arrays[5], having digital-atevery-element transceiver modules allows for dynamic reallocation of the beam forming network as well as the transmission of separate frequencies at each element. Therefore, a given array can be separated into different sections, ideally operating independently of each other as long as the transmit pulses are not saturating the receiving elements operating at a different frequency. A comparison of a side by side split of an array with an interleaved split of the array (see Figure 1) is given to show the possibilities of concurrent transmission and reception.

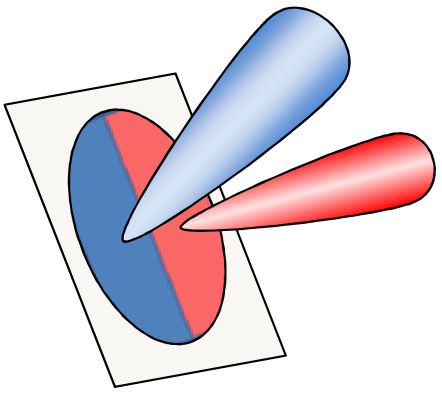

(a)

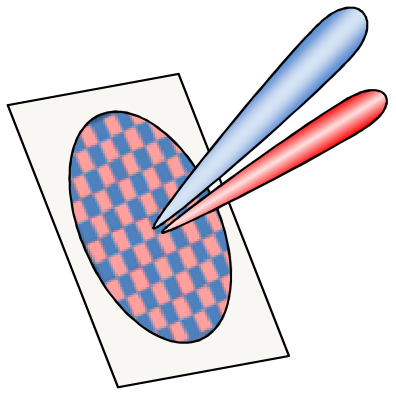

(b)
Figure 1 - (a) Array face with contiguous regions operating at separate frequencies, (b) Array with interleaved elements at different frequencies.

The phased array configuration depicted in Figure 2 is of two arrays which are interleaved. The arrays are interleaved triangular lattices with a cell shape that is closer to a right isosceles triangle than equilateral triangle. This forms the familiar "checker board" pattern. Each element has a notch filter to isolate the receive chain from the coupled transmitter energy from adjacent elements. The effect of this mutual coupling will change as a function of scan angle and the required notch filter depth therefore is complicated. It is instructive to know the required depth of the filter to ensure that a practical realization is possible.

In systems that are spectrally aware of their environment, components are required that enable flexible operation over wide bandwidths. These components must quickly adapt their frequency, modulation, and block out interference from

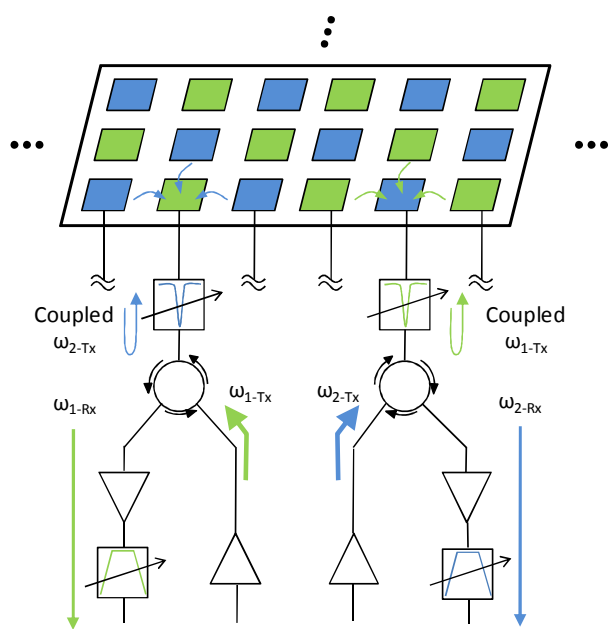

Figure 2 - Example of an interleaved phased array operating at different frequencies at every other element.

outside sources. For example, recent developments through the DARPA ASP program have led to the advent of high-Q widely tunable filters. Both electrically tunable notches and bandpass filters have been developed. Cascades of these components have shown very high isolation, such as in Figure 3. High-Q tunable filters enable these systems to operate in a crowed electromagnetic environment [6]. Using widely tunable high-Q filters with a phased array system that is digital at every element enables the system to isolate itself from co-site interference between neighboring elements while retaining frequency agility on receive and transmit to match filtering capabilities.

High Isolation Tunable Fitlers - The tunable filter configuration used in this analysis was a bandpass-bandstop tunable cascade, described in [7]. Both the notch and bandpass filters are comprised of substrate integrated cavities that are electrically tuned by actuating piezoelectric discs. The filters can tune independently of each other, and by itself, the notch can provide $30 \mathrm{~dB}$ of isolation with $1 \mathrm{MHz}$ bandwidth and $40 \mathrm{~dB}$ peak. The bandpass filter exhibits good roll-off characteristics, and when cascaded together, the bandstop-bandpass combination provides better than $90 \mathrm{~dB}$ of isolation between the pass-band and stop-band at less than $75 \mathrm{MHz}$ separation over $2.5-4 \mathrm{GHz}$, with an insertion loss of $3 \mathrm{~dB}$ in the pass-band. S-parameters are shown in Figure 3 for one tuning of the bandstopbandpass combination. For concurrent transmission on $\omega_{1}$ while receiving on $\omega_{2}$, the notch filter would be centered on $\omega_{1}$ while the bandpass filter is centered on $\omega_{2}$, with the goal of reducing $\omega_{1}$ enough such that it does not saturate any components in the receiver chain. If greater isolation from the transmitted $\omega_{1}$ frequency is needed on the receiver, $\omega_{1}$ and $\omega_{2}$ can be separated further in frequency such that $\omega_{1}$ falls on where the bandpass filter out-of-band rejection is higher. Alternatively for lower isolation, the $\omega_{1}$ and $\omega_{2}$ can be closer in frequency, or even the notch by itself could be used, if less than $40 \mathrm{~dB}$ isolation is required. 


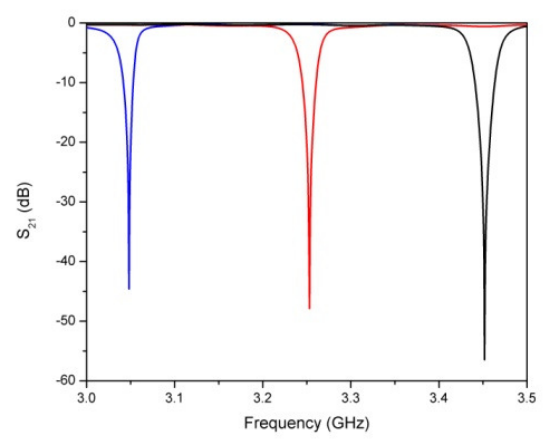

(a)

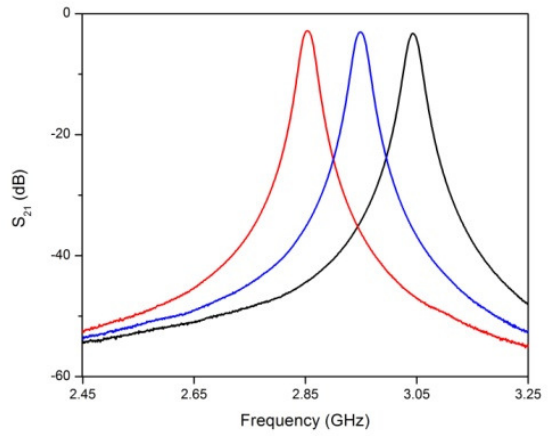

(b)

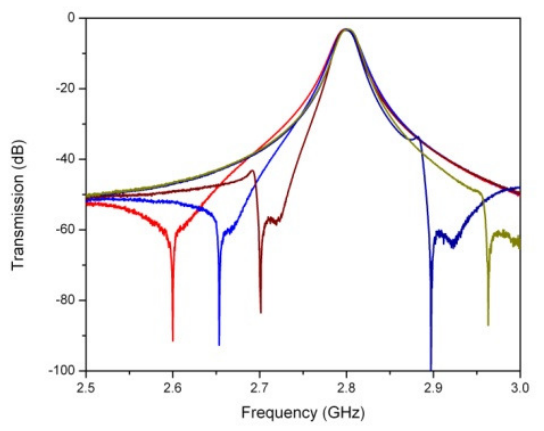

(c)

Figure 3 - Example S-parameters of tunable filters: (a) Bandstop filter, (b) Bandpass filter, (c) Bandstop-bandpass cascade.

Receiver dynamic range - To avoid impacting noise figure, this cascade can be separated such that the bandpass section follows the front end low-noise amplifier (LNA), as in Figure 2. This requires that the notch has high enough isolation to drive down the coupled power at least below the P1dB point of the LNA and preferably down to the peak input power of the spur-free dynamic range (SFDR) for the LNA. GaN LNAs have been demonstrated at $2 \mathrm{~W}$ with an output third-order intercept point (OIP3) of $45 \mathrm{dBm}$ between $3-4 \mathrm{GHz}$ and a noise figure of $0.8 \mathrm{~dB}[8]$. The high dynamic range of this monolithic microwave integrated circuit (MMIC) design reduces the burden on the notch to suppress interference below required levels, or allows for higher transmit power in this configuration. For very high levels of coupled power, the cascaded bandpass-bandstop combination can be inserted before the LNA for additional isolation at the expense of increased noise figure.

Using these LNA and filter parameters, the notch and LNA combination was analyzed to approximate what level of coupled power from adjacent transmitting elements was acceptable. At $3.2 \mathrm{GHz}$, the gain of the LNA in [8] is approximately $14 \mathrm{~dB}$, which makes the input IP3 point $31 \mathrm{dBm}$. Assuming a noise floor of $-110 \mathrm{dBm}$ and knowing that the bandpass filter will reduce the noise-bandwidth after the LNA, the SFDR is as follows in Equation (1).

$$
\begin{aligned}
\text { SFDR } & =\frac{2}{3}\left(P_{3}-N_{0}\right) \\
& =\frac{2}{3}(31 d B m-(-110 d B m))=94 d B m
\end{aligned}
$$

Given this, the maximum input to the LNA to avoid third order products above the noise floor is $-16 \mathrm{dBm}$. With $30 \mathrm{~dB}$ of isolation from the notch preceding the LNA, coupled interference levels from transmitting elements can be as high as $14 \mathrm{dBm}$ before $3 \mathrm{rd}$ order distortion products show up. Depending on the transmit and receive waveform bandwidth, third order interference products due to the transmitter will not spill over into the receive band given enough separation between transmit and receive bands. The real driver of dynamic range in a phased array with digital elements is the analog-to-digital converter (ADC). SFDR values of $80 \mathrm{~dB}$ are common for commercially available
ADCs. With $80 \mathrm{~dB}$ as a guideline for system SFDR and leaving $10 \mathrm{~dB}$ headroom for the minimum detectable signal, $70 \mathrm{~dB}$ was targeted as the required maximum difference between the desired receive signal and interfering transmitted signal at the input to the ADC. Using these figures, the coupled interference from transmitting elements on the phased array was determined versus scan angle and ultimately the allowable transmitter power with this dynamic range requirement with the bandstop-bandpass filter combination on the receiver for isolation.

\section{ARRAy Simulation Results}

To determine the coupled power into a phased array element from adjacent elements operating at different frequencies, an $8 \times 8$ stacked patch microstrip array was characterized in an anechoic chamber[9]. S-parameter measurements were taken between one of the center elements and all other elements. The coupling factor, $\mathrm{P}_{\mathrm{A}}$, into an element $\mathrm{A}$ is the addition of the all the $S_{\mathrm{jA}}$ parameters in Equation (2),

$$
P_{A}=\sum_{1}^{N} S_{j A} e^{i k_{0}\left(\left(x_{j}-x_{A}\right) \sin \theta \cos \phi+\left(y_{j}-y_{A}\right) \sin \phi\right)}
$$

where $\theta$ and $\phi$ are the elevation/azimuth scan angles, $x_{j}$ and $\mathrm{y}_{\mathrm{j}}$ are the positions of the $j$-th element on the array, and $\mathrm{N}$ is the number of elements. The exponential factor applies a phase shift that scans the array in the $(\theta, \phi)$ direction. To determine coupled power levels, for example, suppose each transmitting element is supplied with $10 \mathrm{~W}$ and $\mathrm{P}_{\mathrm{A}}$ is $-10 \mathrm{~dB}$, then the interfering power into element $\mathrm{A}$ is $1 \mathrm{~W}$.

Split contiguous arrays - In this concept, large sections of the phased array antenna face are assigned to operate at different frequencies. These could be comprised of small groups of elements up to separate halves of the full array. For concurrent transmission while receiving on adjacent sections of the full array, interference at the boundaries between these sections will be the most problematic, and the level of coupling is dependent on the scanning angle of the transmitting array section. Coupled power levels in the split contiguous array were analyzed for different scan angles. As depicted in Figure 4, Array 1 was the transmitting array, while Array 2 was receiving at a separate frequency. 


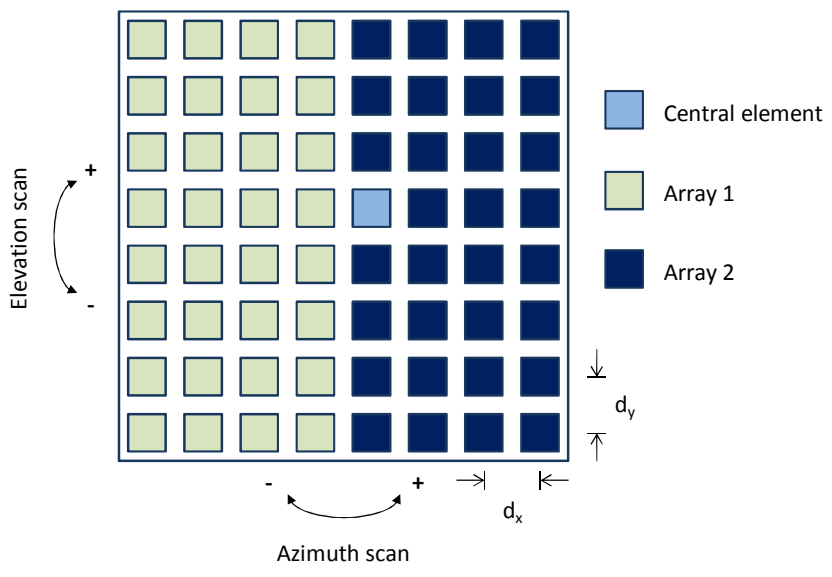

Figure 4 - Split contiguous array configuration at two different frequencies. Interference from Array 1 and active $S_{11}$ parameters were calculated using Array 2 relative to the central element at different scan angles.

The coupling factor, $\mathrm{P}_{\mathrm{A}}$, from Array 1 to the central element was calculated for various scan angles of Array 1 at $3.3 \mathrm{GHz}$ and is plotted in Figure 5a. There are some scan angles with much lower coupling than other angles, with as much as $10 \mathrm{~dB}$ variance. Active $S_{11}$ was also examined with this array configuration, treating Array 2 and the central element as the transmitting array. Over the $\pm 50^{\circ}$ azimuth/elevation scanning range, active $S_{11}$ was better than $-6 \mathrm{~dB}$ at $3.3 \mathrm{GHz}$ at peripheral scan angles and better than $-10 \mathrm{~dB}$ for all other angles.

Effect of Frequency versus scan angle - Since the filtering can be tuned across the band, it is of interest to determine the impact of optimizing the frequency for a given scan angle to minimize the amount of coupling. Coupled interference from transmitting elements to receiving elements can be reduced by changing the transmit frequency for a given scan angle. The coupling factor from Equation (2) was calculated for the same scanning range over 3$3.8 \mathrm{GHz}$ and the minimum coupling factor versus scan angle in this frequency range is shown in Figure 5b. Compared to the non-optimized single frequency coupling versus scan angle in Figure 5a, coupling decreases $0-6 \mathrm{~dB}$ for most scan angles with one region decreasing 20dB. Over all, the average coupling for the single frequency scan is $-14.3 \mathrm{~dB}$, where the coupling for the optimized multi-frequency scan is $-17.3 \mathrm{~dB}-\mathrm{a} 3 \mathrm{~dB}$ average improvement.

The optimal frequencies at which the minimum coupling values were found are shown in Figure 6, which gives an idea of how often a phased array system would have to change its transmit frequency as it scans electronically. Of note, there are large sections of scan angles that have the same optimal frequency for minimum coupling. To operate two separate functions concurrently within one of these zones, a compromise must be made by tuning transmit and receive bands slightly off the optimal frequency in opposite directions, far enough such that the required isolation can be provided by the bandstop-bandpass filters.

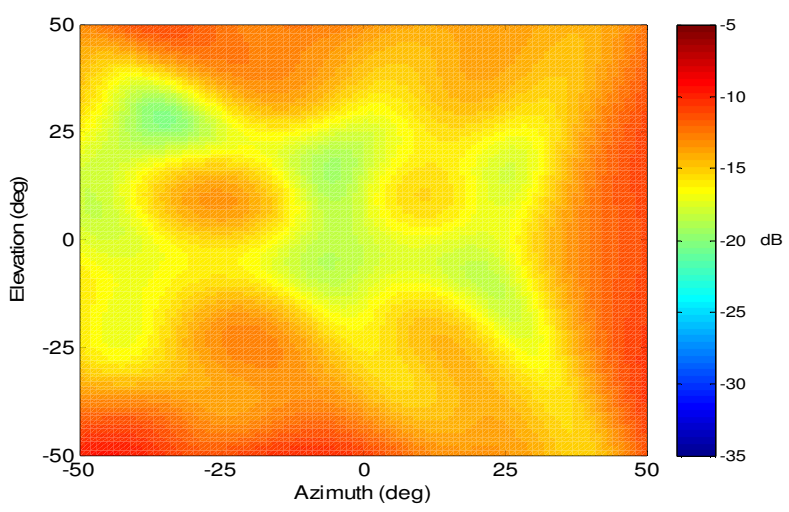

(a)

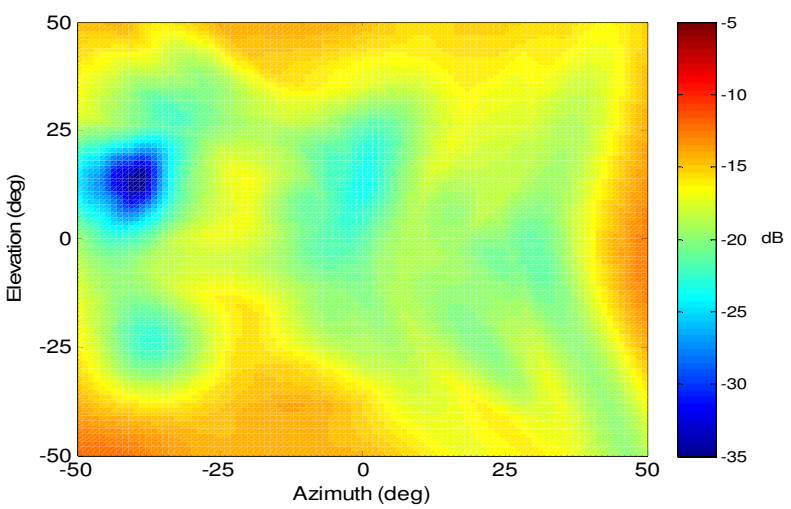

(b)

Figure 5 - (a) Coupling factor from large split contiguous array block of transmitting elements into immediately adjacent central element at $3.3 \mathrm{GHz}$, (b) Minimum coupling factor from Array 1 to central element in Array 2 at the optimal frequency between $3-3.8 \mathrm{GHz}$.

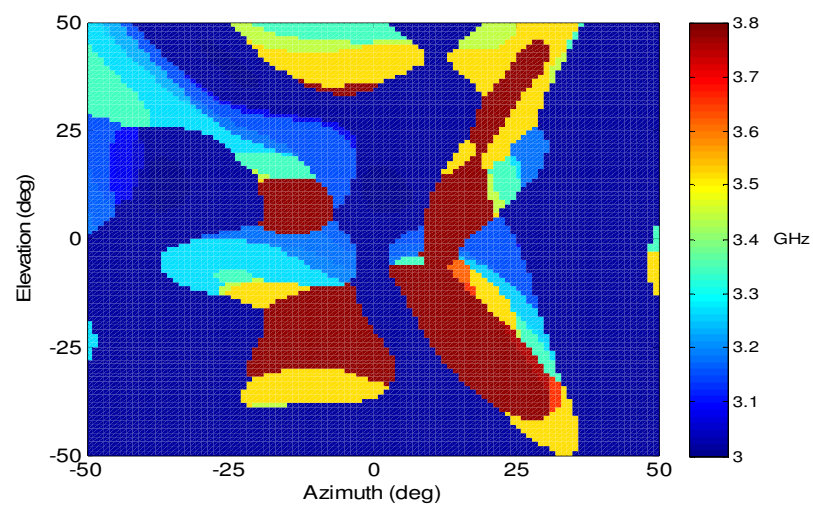

Figure 6 - Associated frequency with minimum coupling value in Figure 5b.

Interleaved arrays - With the split array configuration at two or more different frequencies, beam width is wider than it would have been for the entire array face operating at a single frequency. The elements can be configured as in Figure 7 to increase the effective aperture area at the expense of reduced scanning range due to grating lobe limitations. Tunable filters to protect receiver chains would be configured as in Figure 2. 


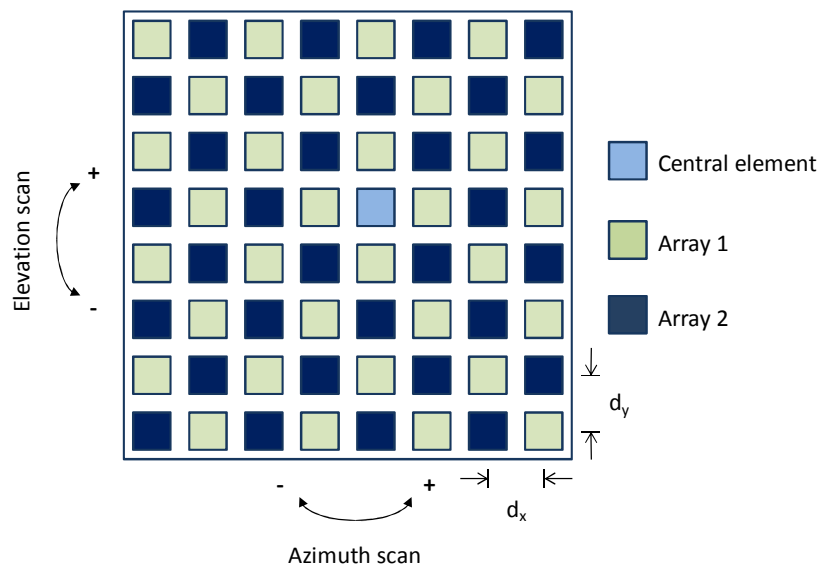

Figure 7 - Interleaved phased array elements at two different frequencies.

For this configuration, active $S_{11}$ was first determined for various scan angles. This $S_{11}$ value is what the central element in sees when it transmits with Array 2. Since Array 1 and Array 2 have the same element spacing, the calculated active $S_{11}$ was used for both arrays and approximates what an element would see in a much larger array.

Noting $S_{11}$ for a given scan angle, minimum values for coupled power into the central element from Array 1 were determined only for where the active $S_{11}$ was better than $6 \mathrm{~dB}$ - where the array was decently matched. The minimum coupled power versus scan angle over $3-3.8 \mathrm{Ghz}$ for the interleaved array is shown in Figure 8.

Around azimuth or elevation only scan directions, the coupled power into the central element from Array 1 ranges between $-7 \mathrm{~dB}$ to $-14 \mathrm{~dB}$. The coupled interference level generally decreases with more oblique scan angles, with coupling factors as low as $-60 \mathrm{~dB}$ at a few select scan angles. With coupling that low, it is conceivable to implement concurrent transmit and receive architectures without the need for the band-stop filter in front of the LNA. However, this does not account for grating lobes, which show up at frequencies above $3 \mathrm{GHz}$ at angles above and to the right of the line in the figure.

Grating lobes - Since the arrays are now sparse in the interleaved configuration, grating lobes show up at smaller angles when scanning off boresight. Grating lobe locations $(\theta, \phi)$ are given by Equation (3),

$$
\begin{aligned}
& \sin \theta \cos \phi=\sin \theta_{0} \cos \phi_{0}+\frac{p \lambda}{2 d_{x}} \\
& \sin \theta \sin \phi=\sin \theta_{0} \sin \phi_{0}+\frac{q \lambda}{2 d_{y}}
\end{aligned}
$$

where $\left(\theta_{0}, \phi_{0}\right)$ is the desired scan angle. In the case of the $8 \times 8$ array used in this analysis, $\mathrm{d}_{\mathrm{x}}=4.75 \mathrm{~cm}$ and $\mathrm{d}_{\mathrm{y}}=$ $4.62 \mathrm{~cm}$. Grating lobes only show up where $\mathrm{p}+\mathrm{q}=0, \pm 2$, \pm 4 , etc., because of the triangular lattice [10]. Taking this

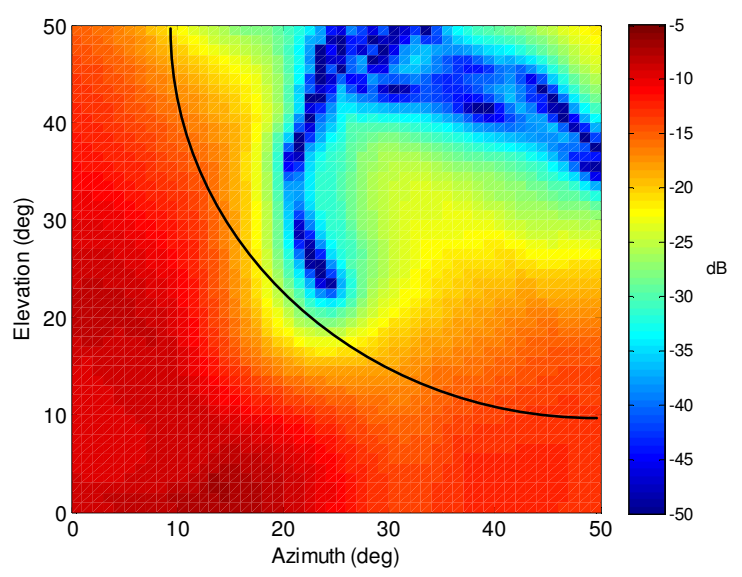

Figure 8 - Minimum coupled power versus scan angle in interleaved array configuration over 3-3.8GHz. Grating lobes occur to the right and above the curve at frequencies above $3 \mathrm{GHz}$.

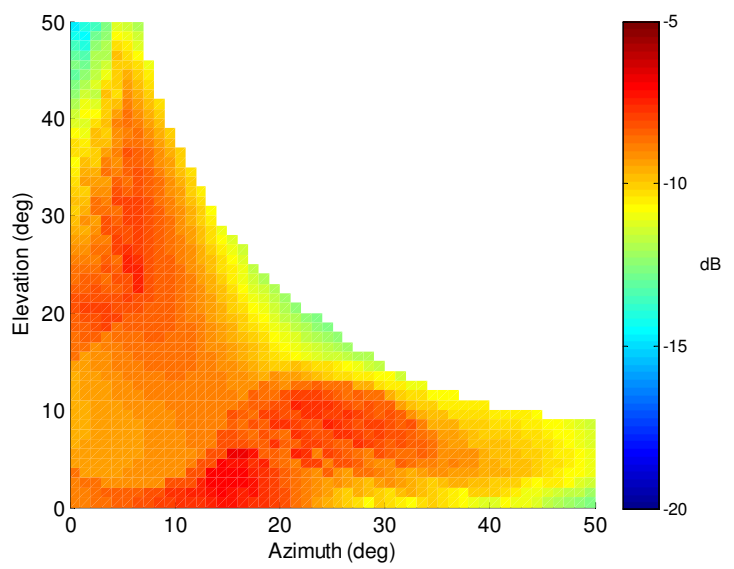

Figure 9 - Minimum coupling factor from transmitting elements to receiving elements at frequencies that do not induce grating lobes.

into consideration, the coupling factor was recalculated while masking off frequencies that induce grating lobes and this result is shown in Figure 9.

The coupling factors still range $-7 \mathrm{~dB}$ to $-14 \mathrm{~dB}$ while avoiding frequencies that induce grating lobes. For the $8 \times 8$ panel, if $10 \mathrm{~W}$ transmitters were on every element, concurrent reception is possible for all scan angles in Figure 9 , given that the system must receive a $-110 \mathrm{dBm}$ signal with the notch, LNA, and bandpass cascade. With a $1 \mathrm{MHz}$ bandwidth signal, the rest of the receiver must have an instantaneous dynamic range of $66 \mathrm{~dB}$ at a point after the bandpass filter further reduces the interfering signal, which is within the $70 \mathrm{~dB}$ range defined earlier based on the ADC. With $25 \mathrm{~W}$ elements, the required dynamic range required goes up to $70 \mathrm{~dB}$, right at the dynamic range limit defined earlier. With the notch filter up front, the LNA is not in danger of becoming saturated due to interference from other array elements. With these design considerations, it is feasible to implement a phased array architecture with concurrent transmit and receive using tunable filters for isolation and frequency diversity at every element to operate 
at frequencies that minimize the amount of coupled transmitter power to receiving elements.

\section{Conclusion}

Based on measured results of an $8 \times 8$ antenna patch array, it was shown that isolation between transmitting and receiving elements can be maximized by selecting an appropriate transmit frequency for each scan angle. In a phased array with two interleaved arrays operating at two separate frequencies, reduced interference from a transmitting element to an adjacent receiving element by is achieved by paring this minimized coupling technique with a tunable bandstop-LNA-bandpass cascade. By retuning the notch filter to match the transmission band from adjacent elements and tuning the bandpass filter to the receive band, high isolation is achieved that reduces the coupled interference from transmitting elements to within the typical dynamic range of an ADC. It was shown that concurrent transmission and reception on alternating phased array elements is possible for $25 \mathrm{~W}$ transmitting elements, given $80 \mathrm{~dB}$ of isolation from filters, $7-14 \mathrm{~dB}$ of isolation from optimized mutual coupling, and a receiver dynamic range of $70 \mathrm{~dB}$, although limited in scan angle due to grating lobes.

\section{REFERENCES}

[1] S. Sabatini and M. Tarantino, Multifunction Array Radar: System Design and Analysis. Boston: Artech House, 1994.

[2] E. R. Billam, "Eclipsing Effects with High-Duty-Factor Waveforms in Long-Range Radar," Communications, Radar and Signal Processing, IEEE Proceedings F, vol. 132, no. 7, pp. 598-603, Dec. 1985.

[3] M. Skolnik, Radar Handbook, 3rd ed. New York: McGraw Hill, 2008.
[4] S. Livingston, J. J. Lee, J. Schafner, and R. Loo, "Reconfigurable Interleaved Phased Array Antenna," 6388631, May 14, 2002.

[5] C. Fulton, P. Clough, V. Pai, and W. Chappell, "A digital Array Radar With a Hierarchical System Architecture," Microwave Symposium Digest, 2009. IEEE MTT-S International, Boston, MA, pp. 89-92, June 7-12, 2009.

[6] H. Joshi, H. H. Sigmarsson, Sungwook Moon, D. Peroulis, and W. J. Chappell, "High-Q Fully Reconfigurable Tunable Bandpass Filters," Microwave Theory and Techniques, IEEE Transactions on, vol. 57, no. 12, pp. 3525-3533, Dec. 2009.

[7] E. J. Naglich, J. Lee, D. Peroulis, and W. J. Chappell, "Tunable, Substrate Integrated, High Q Filter Cascade for High Isolation," Microwave Symposium Digest, 2010. MTT '10. IEEE MTT-S International, Anaheim, CA, pp. 1468-1471, May 23-28, 2010.

[8] K. W. Kobayashi et al., "A 2 Watt, Sub-dB Noise Figure GaN MMIC LNA-PA Amplifier with Multioctave Bandwidth from $0.2-8 \mathrm{GHz}, "$ Microwave Symposium, 2007. IEEE/MTT-S International, Honolulu, HI, pp. 619-622, June 3-8, 2007.

[9] C. Fulton and W. Chappell, "Low-cost, Panelized Digital Array Radar Antennas," Microwaves, Communications, Antennas and Electronic Systems, 2008. COMCAS 2008. IEEE International Conference on, Tel-Aviv, Israel, pp. 1-10, May 13-14, 2008.

[10] R. J. Mailloux, Electronically Scanned Arrays. San Rafael, Ca.: Morgan \& Claypool, 2007. 